\title{
Migratory fish bone in the pharynx, a report of 2 cases
}

Shuyi $\mathrm{Xu}^{1}$, Xiaomin $\mathrm{Li}^{2}$, Yue Peng ${ }^{2}$, Jing Yang ${ }^{2}$, Qianxu Liu ${ }^{2}$, Jiefeng Guo ${ }^{2}$, and Zhijian $\mathrm{Yu}^{1}$

${ }^{1}$ Affiliation not available

2Zhuhai City People's Hospital

January 14, 2021

\begin{abstract}
Fish bones have a risk of damaging the mucosa when lodged in the pharynx. Fish bones migrated into surrounding tissues is relatively unusual. We present 2 cases of patients who ingested fish bone, which migrated into retropharyngeal space and hypopharynx. All fish bones were removed without complications.
\end{abstract}

\section{Title page}

\section{Manuscript title:}

Migratory fish bone in the pharynx, a report of 2 cases

\section{A short running title:}

Migratory fish bone in the pharynx

\section{Authors' names and degrees:}

Shuyi Xu, M.D. ${ }^{2}$, Xiaomin Li, B.S. ${ }^{1}$, Yue Peng, M.D. ${ }^{1}$, Jing Yang, B.S. ${ }^{1}$, Qianxu Liu, Ph.D. ${ }^{1}$, Jiefeng Guo, M.D. ${ }^{1}$, Zhijian Yu, Ph.D. ${ }^{1 *}$

\section{Affiliations:}

Department of Otolaryngology, Zhuhai People's Hospital (Zhuhai hospital affiliated with Jinan University), Zhuhai, China

Department of Ophthalmology, Zhuhai People's Hospital, Zhuhai Hospital Affiliated with Jinan University, Zhuhai, China

\section{Funding sources for the study:}

This study was supported by grants from the Cultivation Project of Zhuhai People's Hospital (No. 2019PY22) and Zhuhai Medical Research Fund Project (No. ZH3310200025PJL).

Potential conflict of interest: None provided.

\section{Corresponding author:}

Zhijian Yu, Ph.D., Department of Otolaryngology, Zhuhai People's Hospital (Zhuhai hospital affiliated with Jinan University), No.79 Kangning Road, Xiangzhou District, Zhuhai 519000, Guangdong, China

Tel.: +860756 2157521

Email: yzjent@163.com 


\section{Acknowledgments:}

The authors thank the patients in this case report.

Text

Key words : Fish bone, migratory, pharynx, laryngoscopy.

\section{Introduction}

Fish bones stuck in the pharynx are the most commonly seen emergency cases in otorhinolaryngology practice. A majority of fish bones are straightforward and uncomplicated, which can be easily removed. However, some of cases may pose a significant challenge. Sharp and pointed fish bones may damage mucosa. Migratory fish bones are relatively unusual, however, they may cause severe consequences, such as local infection ${ }^{1}$, large blood vessels rupture ${ }^{2}$, gaslrointestinal perforation ${ }^{2}$. Therefore, effective treatments should be administered as soon as possible.

Here we describe 2 cases of patients who ingested a fish bone while eating, which migrated into retropharyngeal space and hypopharynx. We discuss our experience in treating these migratory fish bones with specific approach for each patient.

\section{Case presentation}

Case 1: migratory fish bone in the retropharyngeal space.

A 62-year-old male patient had suffered from sudden onset pharyngeal pain after he had fish. He visited the local hospital. Fiberoptic laryngoscopy showed that no abnormalities were detected. CT revealed a foreign body lying transversely at the right piriform fossa. Subsequently, painless gastroscopy was performed in order to remove foreign body. Unfortunately, no foreign body was detected. Laryngeal edema occurred during painless gastroscopy, tracheal intubation was performed.

The patient was referred to our hospital after 4 days. After admission, cervical CT was repeated. CT showed that a foreign body was observed in the right retropharyngeal space. The foreign body was observed between cervical vertebra 1 and 2 (Figure 1A, B). Subsequently, he underwent an exploration of the retropharyngeal space and removal of the foreign body under general anesthesia. During the operation. Foreign body cannot be detected in the plane of the second cervical vertebra. Prolonged the longitudinal incision was performed to find the fish bone in the surrounding tissues. Finally, the foreign body was found in the plane of the third cervical vertebra, which was a fish bone. After 1 week of follow-up the patient showed no symptoms of pain or foreign body sensation.

Case 2: migratory fish bone in the hypopharynx.

A 52-year-old man was admitted to our department with throat pain, foreign body sensation, dysphagia, and mild sialorrhea for 3 days after eating a meal of fish. Cervical CT confirmed the presence of a linear radiopaque structure in the left laryngopharynx (Figure 2A, B). Flexible fiberoptic laryngoscopy could not identify a foreign body (Figure 2C). No mucosal lesion was observed. Gastroscope examination were performed to further ascertain whether there was a foreign body in the hypopharynx. A fish bone was seen in the left piriform fossa (Figure 2D). The fish bone pierced from the left piriform fossa into the posterior pharyngeal wall. The object was grabbed with foreign body forceps. After the examination, the patient immediately felt comfortable.

\section{Discussion}

Two cases we described are migratory fish bones in the pharynx. They were hidden in the flexible fiberoptic laryngoscopy. We adopted specific treatment for each patient. Fortunately, every patient had a favorable outcome.

Patients suspected of fish bone impaction usually undergo physical and laryngoscopic examination. A fish bone in the base of tongue or hypopharynx usually requires flexible fiberoptic laryngoscopy for observation. 
However, it is difficult to determine the precise localisation of fish bone in cases with an endoscopically invisible fish bone. Sharp fish bone is prone to damage to the mucosa, and muscle contractions after entering into the pharynx may contribute to the migration ${ }^{4}$. Migratory fish bone is associated with an increased incidence of complications. Therefore, it is important to make a diagnosis correctly. CT scan is considered as the most sensitive modality for detecting ingested fish bones due to it has a sensitivity of $100 \%$ in the soft tissues ${ }^{5}$. Therefore, CT is recommended in cases of suspected migration of ingested foreign bodies into surrounding tissues, as demonstrated in the present cases.

The retropharyngeal space consists of the loose soft tissue between the buccopharyngeal fascia and the prevertebral fascia. Due to the structure of the retropharyngeal space, we surmised that fish bone can migrate freely in the retropharyngeal space. Indeed, we found that fish bone entered the retropharyngeal space can swam away in the first case. During the operation, it is difficult to identify the location of fish bone in the retropharyngeal space. We suggest that locating the approximate position of the fish bone by $\mathrm{CT}$, in addition, surgeons should prolong the longitudinal incision to find it in the surrounding tissues.

The incidence of foreign bodies in the hypopharynx is not uncommon. However, complete visualization of the foreign body in the hypopharynx is usually difficult because most of the hypopharynx is anatomically closed in the resting state. Modified Killian's method can be applied to remove of an endoscopically invisible fish bone $^{5}$. Unfortunately, the second case had severe sore throat symptoms when he bowed and turned head. We removed the fish bone through gastroscopy. During the course of gastroscopy, the hypopharynx was fully exposed. This suggests gastroscopy may be a good alternative method for visualization and removal of fish bones in such cases.

\section{Conclusion}

Cinicians should be aware that fish bone may migrate into surrounding tissues when it cannot be exposed in the pharynx on routine inspection. Early diagnosis of migratory fish bone and therapeutic management are essential for optimal patient survival.

\section{References}

1. Wu E, Huang L, Zhou Y, Zhu X. Migratory Fish Bone in the Thyroid Gland: Case Report and Literature Review. Case Rep Med2018;2018:7345723.

2. Jiang D, Lu Y, Zhang Y, Hu Z, Cheng H. Aortic penetration due to a fish bone: a case report. $J$ Cardiothorac Surg 2020;15:292.

3. Wu CW, Chiu YW. Unintentional fish bone ingestion causing perforation of small intestine. Intern Emerg Med 2020.

4. Ho NH, Chang FC, Wang YF. Clinical Approaches to Migrating Ingested Foreign Bodies in the Neck. Ear Nose Throat J 2020:584320051.

5. Kikuchi D, Ikeda M, Murono S. Endoscopic removal of a fish bone foreign body in the hypopharynx with the modified Killian's method. Auris Nasus Larynx 2020.

\section{Figure legends}

Fig. 1. The fish bone in the retropharyngeal space. Sagittal (A) and axial (B) computed tomography (CT) images showing linear calcification in the retropharyngeal space (red arrow).

Fig. 2. The fish bone in the hypopharynx. Sagittal (A) and axial (B) CT images showing linear calcification in the hypopharynx (red arrow). (C) Flexible fiberoptic laryngoscopy showing no foreign body. (D) Gastroscopy view of a foreign body fish bone in the hypopharynx (yellow arrow). 

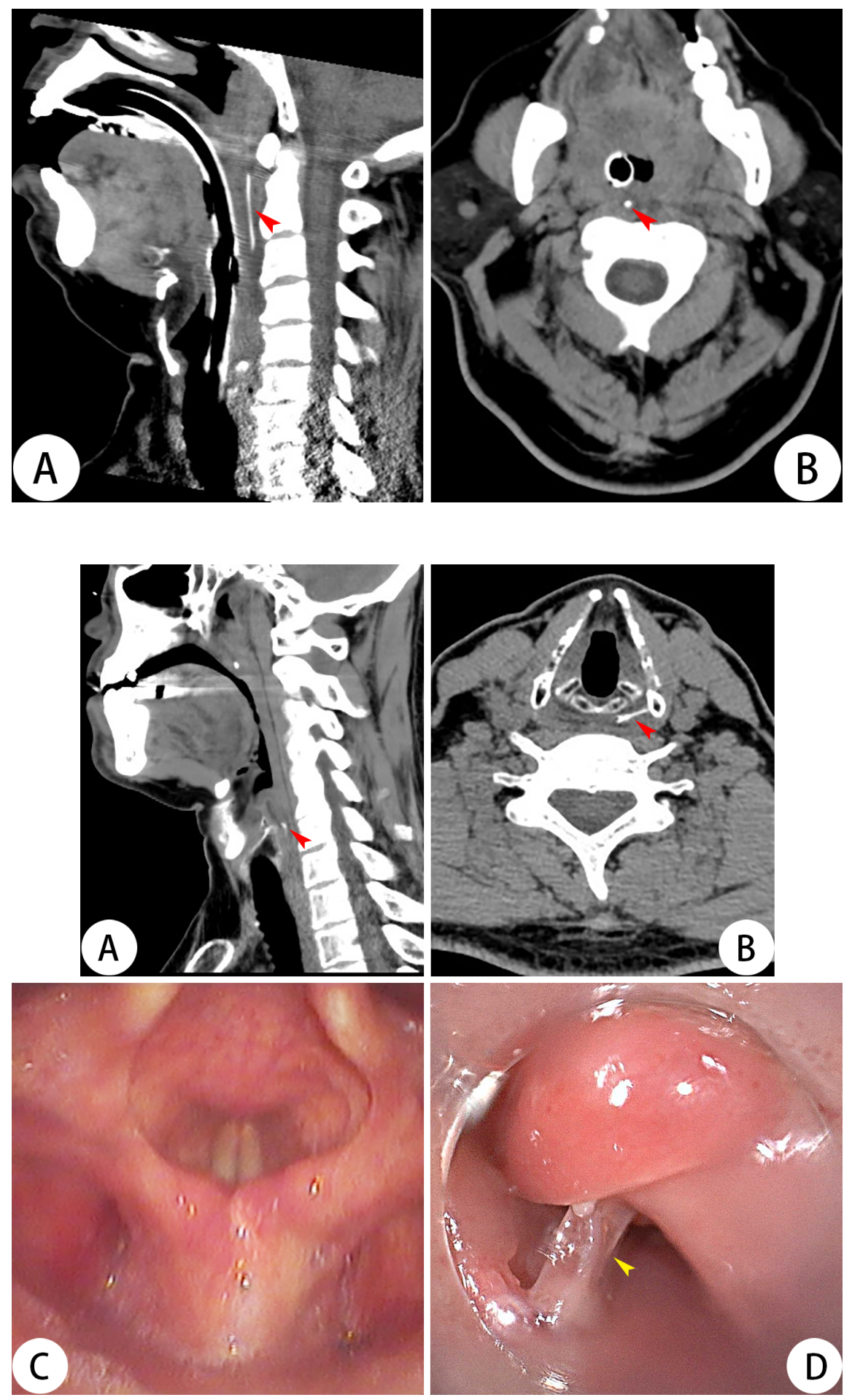